\title{
Quasiparticle Recombination Time in Superconducting Tin and Normal Electronic Density of States at the Fermi Surface from Tunnel Junction Experiments
}

\author{
P.W. Epperlein *, K. Lassmann, and W. Eisenmenger \\ Universität Stuttgart, Physikalisches Institut, Stuttgart, Fed. Rep. Germany \\ Received July 3, 1978
}

\begin{abstract}
We have measured the temperature dependence of the effective quasiparticle recombination time in superconducting tin tunnel junctions by current and laser pulse excitation. The experimental times show satisfactory agreement with calculations based on the ray acoustic lifetime model of Eisenmenger et al. taking into account the film thickness dependence of the phonon reabsorption, $2 \Delta$-phonon volume loss processes and phonon transmission from the junction into the substrate and liquid helium. On the basis of the BCS density of thermally excited quasiparticles and simplified rate equations for quasiparticle recombination, and from the analysis of measurements of decaying excess quasiparticle concentrations we obtain a mean value $N_{0}=(2.73 \pm 0.03) 10^{22} \mathrm{eV}^{-1} \mathrm{~cm}^{-3}$ for the electronic density of states at the Fermi Surface in thin, evaporated tin films. This value differs less than $5 \%$ from that obtained from the experimental electronic heatcapacity coefficient of the bulk material.
\end{abstract}

\section{Introduction}

The minimum energy difference between the ground state of a superconductor at a temperature below the transition temperature $T_{c}$ and the lowest quasiparticle states corresponds with the BCS energy gap $\Delta(T)$. Thermally excited quasiparticles with energies $E \geqq \Delta$ decay to the ground state within a characteristic time $\tau_{R}$ via the formation of Cooper-pairs and the emission of phonons of the energy $\hbar \omega \geqq 2 \Delta$. Whereas $\tau_{R}$, the intrinsic recombination time, is not directly accessible by experiment, an effective time constant $\tau_{\text {exp }}$ can be determined from the decay of an excess quasiparticle concentration generated by an external dynamic creation mechanism, such as pair breaking by the absorption of phonons [1,2] or photons [3-6] or by tunneling between superconductors [7-15]. As has been shown in several experimental $[1-5,10,12-$ $15]$ and theoretical $[10,16,17]$ investigations, $\tau_{\exp }$ significantly exceeds $\tau_{R}$ by the trapping of recombination phonons via repeated reabsorption and

* Future address: IBM Zürich, Research Laboratory, CH-8803 Rüschlikon, Switzerland emission of 2 $2 \Delta$-phonons within the superconducting device. The effectiveness of phonon trapping essentially depends on the sample thickness and the acoustic surface boundary conditions for phonon escape to the adjoining media, i.e. substrate material and liquid helium or vacuum, respectively $[2,15,17]$.

Up to now two different experimental techniques have been applied for the determination of $\tau_{\exp }$, the indirect steady-state $[3,5,7-10,13-15]$ and the direct decay time $[1,2,4,6,10-12]$ measurement. The steady-state method usually measuring $\tau_{\exp }$ in a sandwich-structure of two tunnel junctions requires the knowledge of the density $N_{0}$ of electronic states at the Fermi level as resulting from heat-capacity measurements of the clean bulk material, see e.g. [8]; alternatively, also the band-structure quantity has been used $[10,14]$. The samples for the $\tau$-experiments, in contrast, consist of vacuum-deposited films of microcrystalline structure, usually with high disorder. Consequently, the question arises for the value of $N_{0}$ in disordered metal films. 
We report here on direct measurements of the effective quasiparticle recombination time $\tau_{\text {exp }}$ in superconducting tin and a new experimental technique to determine $N_{0}$ in thin superconductor layers. Using electron tunneling between superconductors for generating and probing an excess quasiparticle density under pulsed conditions, this method has also the advantage of being able to check directly the dependence of $\tau_{\exp }$ on "over-injection". Moreover, we have investigated to what degree $\tau_{\exp }$ differs from quasiparticle excitation by current pulse (excitation energy $E \simeq \Delta$ ) and laserpulse $(E \gg \Delta)$ for the same sample. The measured temperature dependence of $\tau_{\text {exp }}$ is compared with that calculated from a geometrical-acoustic 2 2 -phonon trapping lifetime model [17]. Possible disturbing influences on the exponential shape of $\tau_{\exp }(T)$ at low temperatures are studied.

\section{Theory}

The method to be described is based on the fundamental relationship

$\delta N=N-N_{\text {th }}=I_{0} \tau_{\text {exp }}$,

between the excess quasiparticle density $\delta N$, the stationary injection or generation rate $I_{0}$ and $\tau_{\exp }$. According to Rothwarf and Taylor [16] $\tau_{\exp }$ can be expressed by

$\tau_{\exp }=\left[\left(\frac{N_{\mathrm{th}}}{I_{0}}\right)^{2}+\frac{2 N_{\mathrm{th}}}{I_{0}} \tau_{\mathrm{exp}, 0}\right]^{1 / 2}-\frac{N_{\mathrm{th}}}{I_{0}}$,

where $\tau_{\text {exp }, 0}$ is the experimental recombination lifetime related to $\delta N / N_{\text {th }} \ll 1$ and $N_{\text {th }}$ the equilibrium concentration of thermally excited quasiparticles. The expression

$\tau_{\text {exp }}=\tau_{\text {exp, } 0} /\left(1+\delta N / 2 N_{\text {th }}\right)$

for the dependence of $\tau_{\exp }$ on the overinjection $\delta N / N_{\mathrm{th}}$ is obtained from (1) and (2). With the BCS density of states $N_{\mathrm{tb}}$ is approximated in the temperature range $T<0.5 T_{c}$ by

$N_{\mathrm{th}}=N_{0}\left[2 \pi \Delta(T) k_{B} T\right]^{1 / 2} \exp \left[-\Delta(\mathrm{T}) / \mathrm{k}_{B} T\right]$,

where $N_{0}$ designates the density of electronic states of two spins per unit energy at the Fermi level.

Writing $x(0)$ for the relative change $\delta N(0) / N_{\text {th }}$ of quasiparticle concentration during injection we obtain from (1), (2) and (4) the resulting formula for $N_{0}$

$N_{0}=\frac{2 I_{0} \tau_{\exp , 0} \exp \left[\Delta(T) / k_{B} T\right]}{\left[x^{2}(0)+2 x(0)\right]\left[2 \pi \Delta(T) k_{B} T\right]^{1 / 2}}$.
Thus, we need the absolute values of the measurable quantities $T, \Delta, I_{0}, \tau_{\exp , 0}$ and $x(0)$ for an evaluation of $N_{0}$ from (5). These values can be obtained from a single symmetric superconducting tunneling junction $S_{1} \mid$ oxide $\mid S_{1}$, i.e. $A(T)$ and $T$ from the current (i) - voltage $(U)$ - characteristic and $x(0), \tau_{\exp , 0}$ from the time decay of an excess quasiparticle density.

For the following it is important that the thermal tunneling current $i_{\text {th }}$ for junction voltages $k_{B} T / e<U<2 \Delta / e$ is directly proportional to $N_{\mathrm{th}}$ [18], whereas at the gap-voltage $2 \Delta / e$ additional, nonthermally excited quasiparticles $(E \geqq \Delta)$ are injected into the tunneling volume $V_{T u}$ by breaking up Cooper-pairs. Accordingly we have

$\delta N / N_{\mathrm{th}}=\delta i / i_{\mathrm{th}}$

in the bias regime below $2 \Delta / e$ and

$I_{0}=2 i_{\text {eff }} / e V_{T u}$,

for a sufficiently high current pulse of strength $i_{P}$ driving the voltage of the junction, biased at $U_{B}<2 \Delta / e$, to $2 \Delta / e$. Preconditions for the exactness of (6) are that $\delta i$ and $i_{\text {th }}$ are measured at the same bias and that the excess quasiparticles under stationary injection rate have the same energy distribution as the thermally excited quasiparticles $N_{\mathrm{th}} . \tau_{\mathrm{exp}, 0}$ is directly observed from the time decay of $\delta i(t)$ sufficiently long after switch-off of the injection pulse.

For obtaining the effective excitation current $i_{\text {eff }}$ the thermal single-particle current $i_{\text {th }}$ (at $U=2 \Delta / e$ ), the extra current due to the higher density of quasiparticles $\delta i(0)$, the leakage current $i_{L}$ (at $U=2 \Delta / e$ ) and the so-called two-particle current $i_{2 P}$ [19] (at $U$ $=2 \Delta / e)-($ or excess tunneling current at $U \geqq \Delta / e$ due to contributions of the $A C$-Josephson-effect)-have to be subtracted from $i_{P} \pm i_{B}$, where the positive (negative) sign is valid if pulse current $i_{P}$ and $D C$ current $i_{B}\left(U_{B}\right)$ have the same (opposite) direction. The total injection rate including $i_{2 P}$ as an excitation current is, however,

$$
\begin{aligned}
& I_{0, \text { total }}=2 i_{\text {eff }} / e V_{T u}+i_{2 P} / e V_{T u} \\
& =2\left\{i_{P} \pm i_{B}\left(U_{B}\right)-i_{L}(2 \Delta / e)\right. \\
& \left.\quad-i_{\text {th }}(2 \Delta / e)[x(0)+1]-i_{2 P}(2 \Delta / e) / 2\right\} / e V_{T u} .
\end{aligned}
$$

A simultaneous measurement for $i_{P}$ and $\delta i(0)$, i.e. $\delta i(0) / i_{\text {th }}$ is not possible. However, under certain conditions the nonlinear rate equations for disturbed quasiparticle and phonon systems [16] can be simplified and integrated and thus furnish a nonexponential decay function $\delta N(t) / N_{\mathrm{th}}=x(t)$ after injection, so that, knowing the value of $\delta N / N_{\text {th }}$ for some time $t$, we can reextrapolate to $t=0$ and obtain the value $x(0)$ during the injection. Writing $\dot{x}$ for $d x / d t$ we derive 
from the rate equations the exact expression

$\ddot{x}+\dot{x}\left\{\frac{x+1}{\tau_{R}}+\frac{1}{\tau_{\mathrm{ph}}}+\frac{1}{\tau_{y}}\right\}+\frac{1}{2 \tau_{y} \tau_{R}} x(x+2)=0$.

$\tau_{\mathrm{ph}}$ and $\tau_{\gamma}$ are the lifetimes of a gap-frequency phonon in the superconducting tunneling junction due to reabsorption and processes other than pair excitation respectively, i.e. phonon transmission across the junction surface boundaries and inelastic volume phonon interactions [20].

We substitute $\dot{x}=p$ and $\ddot{x}=p d p / d x$ and insert $\tau_{\text {exp }, 0}$ $=\tau_{\gamma} \tau_{R}\left(\tau_{\mathrm{ph}}^{-1}+\tau_{\gamma}^{-1}\right)$ [16] in Equation (9) and obtain

$\frac{d p}{d x}+\frac{x+1}{\tau_{R}}+\frac{\tau_{\exp , 0}}{\tau_{\gamma} \tau_{R}}+\frac{1}{2 \tau_{y} \tau_{R}} \frac{x(x+2)}{p}=0$.

Neglecting the first and second term this equation can be integrated whence follows:

$x(t)=2\left\{[1+2 / x(0)] \exp \left(t / \tau_{\exp , 0}\right)-1\right\}^{-1}$

or

$x(0) \equiv x(t=0)$

$=2\left\{[1+2 / x(t)] \exp \left(-\mathrm{t} / \tau_{\exp , 0}\right)-1\right\}^{-1}$,

which is the value of $\delta N / N_{\mathrm{th}}$ required in (5). A mean least-squares fit with Equation (11) on the experimental decay curves supplies values for $x(0)$ and $\tau_{\text {exp }, 0}$. Comparing the terms of (9) by using the above approximate solution we find that within its validity range the relation

$\tau_{y}, \tau_{\mathrm{ph}} \ll \tau_{\exp , 0}, \tau_{\boldsymbol{R}}$

is true for $x(0) \leqq 1$. $\tau_{\gamma}$ can be adjusted by a proper choice of the junction thickness $d$ and is generally in the order of the phonons' time of flight through the junction due to their efficient radiation into liquid helium [2], i.e. typically $10^{-10} \mathrm{~s}$ for $d$-values of the order of $10^{3} \AA$. The condition $\tau_{\mathrm{ph}} \ll \tau_{R}, \tau_{\exp , 0}$ can be met by measuring at a sufficiently low temperature, since we obtain from detailed balance consideration of the thermal equilibrium $[16,17]$

$$
\begin{aligned}
& \frac{\tau_{\mathrm{ph}}}{4 \tau_{R}}=\frac{N_{\omega, \mathrm{th}}}{N_{\mathrm{th}}} \\
& \simeq \frac{2 \Delta^{2}\left(2 c_{l}^{3}+c_{t}^{3}\right)}{N_{0}\left(2 \pi^{5}\right)^{1 / 2} c_{l}^{3} c_{t}^{3} \hbar^{3}}\left(\frac{Z^{1 / 2}+Z^{3 / 2}+Z^{5 / 2} / 2}{\exp (1 / Z)}\right),
\end{aligned}
$$

with $Z=k_{B} T / \Delta ; N_{\omega, \text { th }}=$ total density of thermally excited $2 \Delta$-phonons and $c_{l}, c_{t}=$ longitudinal and transverse sound velocity. At $Z=0.16(T=1.1 \mathrm{~K})$ we have $\tau_{R} / \tau_{\mathrm{ph}}=155$ for tin.

Furthermore,

$$
\tau_{\text {exp }, 0}=\tau_{R}\left(1+\tau_{\gamma /} / \tau_{\mathrm{ph}}\right) \geqq \tau_{R} \text {. }
$$

An upper limit of $2 \cdot 10^{-10} \mathrm{~s}$ for $\tau_{\mathrm{ph}}$ follows from measurements of thickness dependence of $\tau_{\text {exp, } 0}$ [17]. Finally, an assumption implicit in the rate equations is that the quasiparticle diffusion length $L_{D}$ is much greater than the junction film thicknesses. From measurements of residual film resistances we have determined the electron mean free path due to elastic scattering in the order of the film thicknesses and then we calculated $L_{D} \simeq 15 \mu$. Consequently, injected quasiparticles are homogeneously distributed by diffusive motion within the junction volume $V_{T u}$ before recombination takes place. We express this by a single value $\delta N$ throughout $V_{T u}$.

\section{Experimental Realization and Data}

The electronic measuring system for generating excess quasiparticles by current- and/or laser pulse excitation and recording the corresponding signal decays is shown in Figure 1 [12]. The injection current is supplied from a fast pulse generator

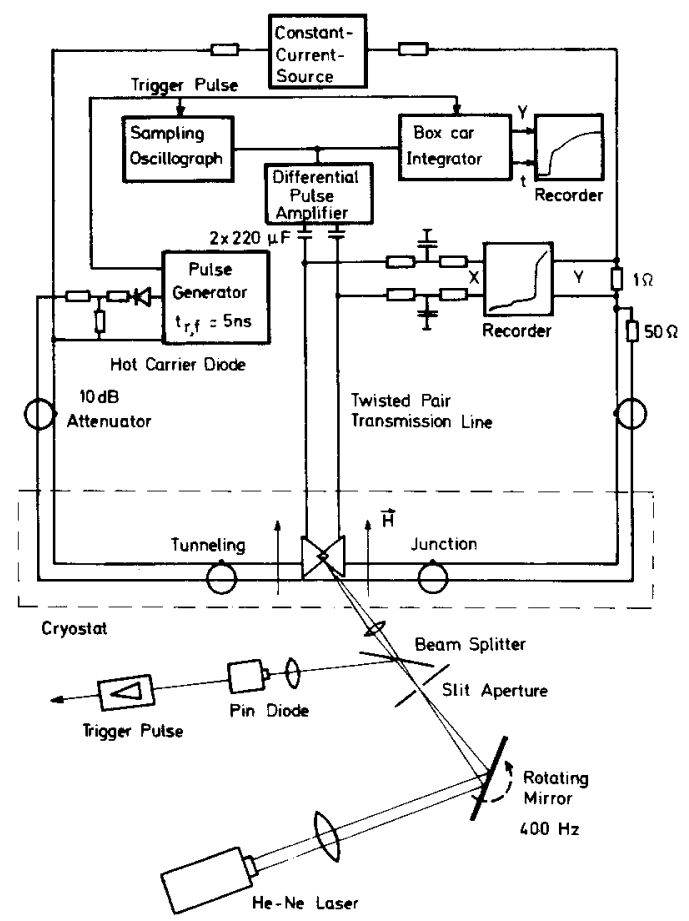

Fig. 1. Schematic diagram of the electrical circuitry for generating excess quasiparticle concentrations in a single superconducting tunnel junction $S_{1} \mid$ oxide $\mid S_{1}$ by current and laser pulses and recording the signals as a function of time. The junction, acting simultaneously as generator and detector is operated at a constant $D C$ bias current. The blocking capacitors $(2 \times 220 \mu \mathrm{F}$, tantalum $)$ of low self-inductance are built in the signal circuit with negligible induction. To avoid any error, the current-voltage characteristics were recorded with open parallel current circuit (inner conductor of the coaxial cable) 
$\left(\tau_{r, f} \simeq 4 \mathrm{~ns}\right)$ connected via a hot carrier diode (Hewlett Packard, Type 5082-2810, $\tau=100 \mathrm{ps}$ ) to a $50 \Omega$-solidjacketed coaxial cable $(0.3 \mathrm{~mm}$ outer diameter $)$, directly leading to the sample and then out of the cryostat. This coaxial transmission line is reflectionfree terminated with a $50 \Omega$-resistor at room temperature. The current terminals of the junction are connected in series with both the ends of the interrupted outer conductor of this cable (see also Fig. 3). The influence of the asymptotic junction tunneling resistance $R_{\infty}$ (typically in the $\mathrm{m} \Omega$-range) on the shape of the primary current pulse can be neglected. The hot carrier diode reduces the time constant of the current pulse and, moreover, suppresses any existing residual ripple. Thus, the switch-off time of the injection pulse is sufficiently well defined. This is important for an exact determination of $x(0)$. As to our junctions this switch-off decay time was about $10 \mathrm{~ns}$. In addition, a small superimposed $D C$-current serves to bias the junction below $2 \Delta / e$ after the switch-off of the current pulse (point 1 in Fig. 2). Since the junction is now operated at a constant $D C$-current, instead of the decaying excess thermal tunneling current $\delta i(t)$, a time dependent voltage $\delta U(t)$ is measured according to

$\delta U(t)=-\delta i(t) \quad\left(R_{d} \| R_{L}\right)$.

$R_{d}$, typically in the $1 \Omega$-range for the used junctions, is the dynamic resistance at the biasing point $U_{B}$ and $R_{L}$ the load resistance of the voltage measuring circuit.

The technique for generating sufficiently fast $\left(\tau_{r, f}<100 \mathrm{~ns}\right)$ light pulses is fundamentally simple and shown in the lower part of Figure 1. The light of a $15 \mathrm{~mW}-\mathrm{He}-\mathrm{Ne}$ laser is focused in the plane of a slit aperture after the reflection at a mirror. By rotating the mirror the reflected laser beam is mechanically chopped at the fixed aperture. The outgoing laser pulses with typical time constants of the leading and trailing edge and half-widths in the order of $50 \mathrm{~ns}$ are focused through the walls of a glass cryostat on the front of the junction. To avoid lattice heating effects in the quasiparticle photoexcitation experiments we used, according to [3], junctions prepared on sapphire substrates which were directly immersed into liquid helium II. Furthermore, although the quasiparticles are produced within the front junction layer in an optical penetration depth of a few hundred angströms, the excess quasiparticle concentration will be approximately uniform throughout the tunneling volume because of the large quasiparticle diffusion length and the indirect action of the recombination phonons.

A symmetrical, twisted pair transmission line (see also Fig. 3) connects directly the voltage terminals of the junctions with the input of a fast differential pulse

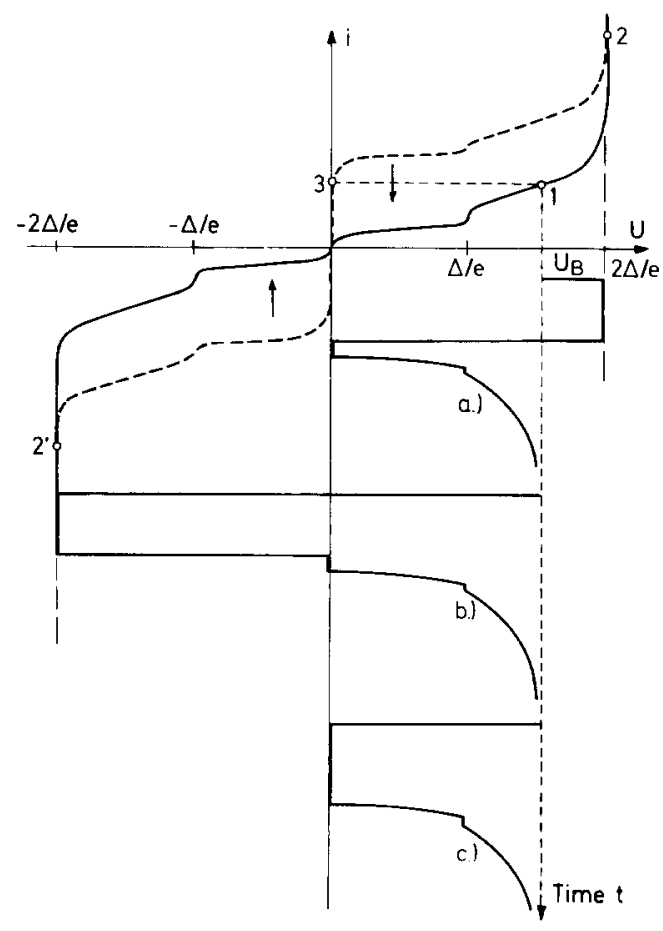

Fig. 2. Schematic drawing of the formation of the time dependent signals due to quasiparticle excitations by current $(\mathbf{a}, \mathbf{b})$ and laser pulses (c) taking account of the constant $D C$-current operation. Point $1 \cong$ bias voltage without injection, point $2 \cong$ biasing point shifted to the gap voltage due to the superimposed pulse current, point $3 \cong$ biasing point shifted to lower voltages due to the excess quasiparticle concentration after the switch-off time of the injection pulse

amplifier. The characteristic line impedance $Z \simeq 100 \Omega$ has been carefully matched to the input resistance $R_{a}=R_{L}=2 \times 50 \Omega$ of the amplifier and inductive geometries on the voltage side could be largely avoided. The amplified voltage signal is sampled and filtered in a PAR boxcar integrator (band width $50 \mathrm{MHz}$, gate width minimum $10 \mathrm{~ns}$ ) and then displayed by $X$-Y-recording as a function of time.

The time dependent shapes of the voltage signals to be expected with quasiparticle injection by current and laser pulses are schematically illustrated in Figure 2. The detailed shapes can be explained by the highly nonlinear $i-U$-characteristics in consideration of the constant $D C$-current operation and the quasiparticle density dependence of $\tau_{\exp }$ [compare (3)]; e.g. the kink in the trailing edge of the voltage pulse corresponds to the current step at $U=\Delta / e$ in the tunneling characteristic. Generally speaking, a convex (concave) curvature of the characteristics as near to the bias level $U=0(U=\Delta / e$ and $2 \Delta / e)$ results in an increase (a decrease) of the real $\tau_{\text {exp }}$.

Provided, however, that the dynamic resistance $R_{d}$ is constant and independent of temperature in the operating region of the junction characteristic, the 
voltage signal $\delta U(t)$ will be proportional to $\delta i(t)$ and thus to $\delta N(t)$. We, therefore, measured the voltage dependence of $R_{d}$ for various temperatures and found out that $R_{d}$ changed approximately to $1 \%$ in the voltage range $0.675 \mathrm{mV} \leqq U_{B} \leqq 0.825 \mathrm{mV}$ and within temperature intervals of $0.1 \mathrm{~K}$ for the used tin-junctions. Consequently, we have chosen this linear operating region with a bias voltage of $U_{B}$ $=0.8 \mathrm{mV} \simeq 0.7 \Delta$. In order to avoid switching from the biasing point $U_{B}$ to the zero voltage state due to the $D C$-Josephson effect, the junctions were operated in a parallel magnetic field of 10 to $25 \mathrm{Oe}$. In the linear regime $\tau_{\exp }$ is directly obtained from the time decay of the voltage signal and is only a function of the momentary overinjection $\delta N(t) / N_{\mathrm{tb}}$. The pulse receiving system has been calibrated in terms of $\delta i$ by means of small, simulated signal currents of known amplitude.

To determine the effective quasiparticle recombination times without any corrections over a wide range, the electronic time constant $\tau_{\text {syst }}$ of the receiving system must be small compared with $\tau_{\exp , 0}$. By analyzing our circuit we obtained for instance $\tau_{\text {syst }}$ $=30 \mathrm{~ns}$ at the biasing point $U_{B}=0.8 \mathrm{mV}$ with $R_{d} \simeq 2 \Omega$. As simulation experiments showed, this time constant is due to the time constant ( $25 \mathrm{~ns}$ ) of the electronic measuring system and to the effective $R C$ time of the junction

$\tau_{T u}=C_{T u} R_{d} R_{L} /\left(R_{L}+R_{d}\right)$.

$C_{T u}$, the capacitance of the tunnel junction, amounts to appr. $25 \mathrm{nF} / \mathrm{mm}^{2}$ tunneling area. By using the parallel plate model for the junction capacitance and assuming $15 \AA$ for the barrier thickness, we obtained for the dielectric constant of the Sn-oxide a value of 4 in good agreement with other tunneling experiments carried out, however, at $\mathrm{Pb}$-samples [21,22]. For any necessary consideration of $\tau_{\text {syst }}$ the measured signal time constants $\tau_{\text {meas, } 0}$ were corrected according the formula

$\tau_{\text {exp }, 0}^{2}=\tau_{\text {meas }, 0}^{2}-\tau_{\text {syst }}^{2}$.

Figure 3 shows the sample configuration used in our experiments. The tunnel junction is formed between two superconducting films elsewhere insulated by an approximately $0.3 \mu$ thick $\mathrm{SiO}$ layer. Thus, the conductor loops in the region of the tunneling area can be made small; the nominal area is about 0.5 $\times 0.6 \mathrm{~mm}^{2}$. The inductive crosstalk from the high injection current pulse to the voltage terminals is, as an estimate showed, essentially determined by the ratio of the SiO-thickness to the width of the junction and amounts to less than $10^{-3}$. At the narrow ends of the sample, the films form a pair of tines, respectively.

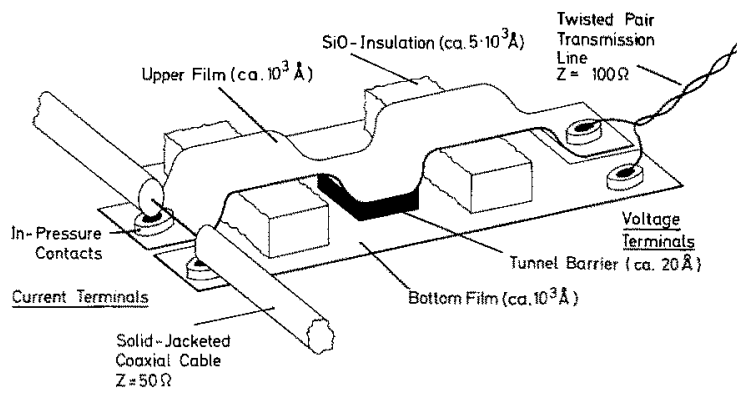

Fig. 3. Tunnel junction configuration for generating and detecting excess quasiparticles with low inductive crosstalk from the current pulse to the voltage terminals. The junction is vacuum-đeposited on a sapphire or silicon substrate (not shown)

with an unshielded width of $0.1 \mathrm{~mm}$. To minimize conductor loops at the leads, contact to the sample is made by pressing the current and voltage cable over the corresponding tines. Decay curves, experimentally obtained with this wide-band junction configuration, are free of disturbing, crosstalk-induced oscillations.

The samples were prepared in the conventional manner by using evaporation techniques. The films were vacuum deposited on a sapphire or silicon substrate under a residual gas pressure of $10^{-6}$ Torr and at a rate of $2 \AA / \mathrm{s}$ to $200 \AA / \mathrm{s}$. Deposition in an oxygen background of about $10^{-5}$ Torr improves the junction quality, defined by the ratio $i_{2 \Delta_{+}} / i_{2 \Delta_{-}}$of the maximal and minimal single particle current at $U$ $=2 \mathrm{~A} / \mathrm{e}$. This current ratio is approximately equal to the resistance ratio $R_{d} / R_{\infty} ; R_{d}$ below $U<2 \Delta / e$ is mainly determined by the leakage current. High $R_{d} / R_{\infty}$-values are important for the signal to noise ratio being proportional to $R_{d} / R_{\infty}$ in this measuring method with high ohmic termination $R_{L} \geqslant R_{d}$. The junction thicknesses $d$ were typically $4000 \AA$ to $5000 \AA$. The tunneling barrier was formed by oxidizing the bottom film in an oxygen glow discharge for 5 min at a pressure of 0.08 Torr. We found out, that the junction quality doesn't depend on the sequence of the processes $\mathrm{SiO}$-evaporation and $\mathrm{Sn}$-oxidation. To prevent any condensation of $\mathrm{SiO}$ in the tunneling area, besides other precautions the distance between the corresponding evaporation mask and the bottom film was chosen as small as possible ( $\leqq 0.1 \mathrm{~mm})$. The obtained low-ohmic samples $\left(R_{\infty}\right.$ in the $1 \mathrm{~m} \Omega$-range) with high quality $\left(i_{2 \Delta_{+}} / i_{2 \Delta_{-}}>200\right)$ indicated that the junction window wasn't coated with any disturbing $\mathrm{SiO}$ layer.

\section{Experimental Results and Conclusions}

Figure 4 shows several time-dependent signal shapes, measured at low temperatures with the described technique in a slightly oxygen-doped (evaporation 


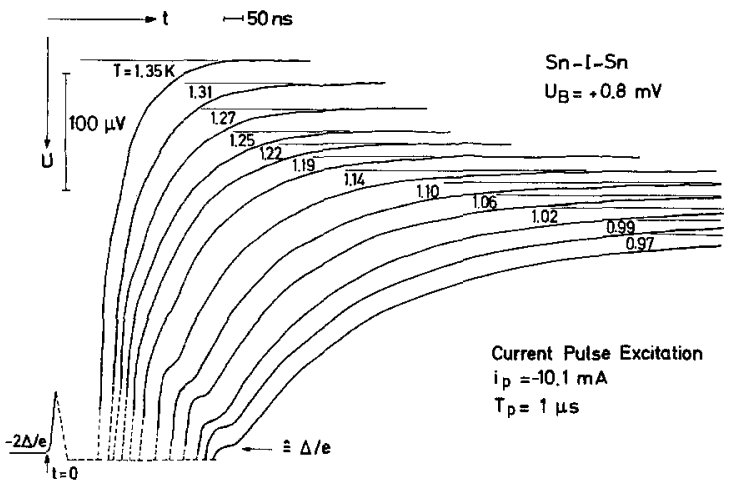

Fig. 4. Time-dependent voltage signal shapes in the linear regime of the $i-U$-characteristics at various temperatures. The curves are shifted vertically each other. The baselines correspond to $U_{B}$ (biasing point 1 in Fig. 2)

pressure $8 \cdot 10^{-6}$ Torr $\mathrm{O}_{2}$ ) tin junction under quasiparticle excitation by current pulses. The junction was biased in the linear range of the tunneling characteristics at $U_{B}=0.8 \mathrm{mV}>\Delta / e$. Current pulses and bias current were opposite in polarity. In Figure 4 we have displayed with high sensitivity only that part of the total voltage signal decays $\left(2 \Delta / e+U_{B}\right)$, ranging from approximately $\Delta / e$ to the baseline $\left(U_{B}=0.8 \mathrm{mV}\right)$. The distinct kink in the various signal shapes is due to the relatively high excess $D C$-current $i_{2 P}=0.25 \mathrm{~mA}$ at $\Delta / e$ in this sample (compare Fig. 2). To determine the switch-off time $t=0$ of the injection pulse, the signals were recorded with zero shift. The logarithmic diagrams of the displayed voltage amplitudes vs. time (not shown) result in straight lines at high temperatures $(>1.10 \mathrm{~K})$, while at low temperatures and high amplitudes (short times) the diagrams deviate from straight lines to shapes with greater slopes according to the effective overinjection. The slopes at sufficiently long times correspond to $\tau_{\exp , 0}$ and agree with the time constants resulting from the fits of Equation (11) on the experimental decay curves. The differential time constants $\tau_{\text {exp }}$, determined from the logarithmic diagrams at any overinjection are in agreement with Equation (3). Moreover, the signals shown in Figure 4 are independent of the pulse polarity; the signals obtained with negative or positive polarities and same effective injection rates differ less than $1 \%$. Hence it follows an undisturbed symmetry of the used electronic measuring system.

The time constants $\tau_{\text {exp }, 0}$, measured from various runs with the same sample under current and laserpulse excitations are plotted as a function of temperature in Figure 5 with the following results:

1) The exponential $\exp \left(\Delta(T) / k_{B} T\right)$ temperature dependence required by theory is verified by the $\tau_{\exp , 0}$ values within $0.95 \mathrm{~K} \leqq T \leqq 1.50 \mathrm{~K}$; at these temperatures the relation $\Delta(T) \simeq \Delta(T=0) \equiv \Delta_{0}$ is valid for tin with a measured maximal difference of $2 \%$. The influence of $\tau_{\text {system }}=30 \mathrm{~ns}$ has been taken into account, compare Equation (18). Consequently, these time constants can be attributed to the effective quasiparticle recombination lifetimes in the tin tunnel junction.

2) The times measured below $T=1.07 \mathrm{~K}$ are corrected according to Equation (3) for the concentration dependence of $\tau$, since additional quasiparticles are injected stationarily at the biasing point $U_{B}$ $=0.8 \mathrm{mV}>\Delta / e$ by the excess, "two-particle" current $i_{2 p}$. This injection reduces $\tau_{\exp , 0}$ to a saturation value at decreasing temperatures. The overinjection $\delta N\left(i_{2 P}\right) / N_{\text {th }}$ was determined from (1) and (2) and with the rate $I_{0}=i_{2 P} / e V_{T u}$. With regard to this overinjection the lifetimes comply with the correct $\exp \left(\Delta_{0} / k_{B} T\right)$-dependence at low temperatures too. Measurements on other tin samples with smaller $i_{2 P}$ showed a correspondingly reduced overinjection. At bias voltages $U_{B}<\Delta / e$ these lifetime corrections were not necessary. Thus, $i_{2 P}$ has to be considered as an injection current with the rate $i_{2 P} / e V_{T u}$.

3) $\tau_{\text {exp }, 0}$ is independent of quasiparticle excitations by current and laser pulses, respectively, within the accuracy in measurement of $\leqq 10 \%$.

4) Any magnetic flux trapped in the superconducting films, probably in form of quantized flux lines (vortices), effects an evident $\tau_{\text {exp }, 0}$ saturation at low temperatures (symbol (•) in Fig. 5). Responsible for this effect is a reduced reemission and reabsorption of $2 \Delta$ phonons in the "vortices-doped" films with a strongly varying energy gap. Furthermore, the $i-U$ characteristics in the thermal tunneling range were shifted to higher currents with increasing voltage, e.g. by $8 \%$ of the total current at $U_{B}=0.8 \mathrm{mV}$ and $T=1 \mathrm{~K}$. In Figure 5, the flux trapping was caused by the geomagnetic field acting perpendicularly on the tunneling area with a strength of only $0.2 \mathrm{G}$ (measured with a Hall Sonde) when cooling down below $T_{c}$. In order to exclude largely these disturbing effects, the tunneling barrier was oriented parallel to the geomagnetic field.

5) Over the temperature range of our measurements, the effective quasiparticle recombination lifetime in the $4200 \AA$ thick superconducting tin tunnel junction on a sapphire substrate immersed in $\mathrm{He}$ II is

$\tau_{\exp , 0}=\tau_{0} \frac{T^{-1 / 2}}{\left[K^{-1 / 2}\right]} \exp \left(\Delta_{0} / k_{B} T\right)$

with $\tau_{0} \simeq 0.6 \mathrm{~ns}$. This value is compared with theory based on a ray-acoustic $2 \Delta$-phonon trapping lifetime model [17] showing a linear thickness dependence of $\tau_{\text {exp }, 0}$ for the relations $d>A_{w}$ and $\Lambda_{v} \gg A_{w}$ between the mean free paths of $2 \Delta$-phonons due to reabsorption $\left(A_{w}\right)$ and bulk loss processes $\left(\Lambda_{v}\right) ; \Lambda_{w} \leqq 700 \AA$ in 


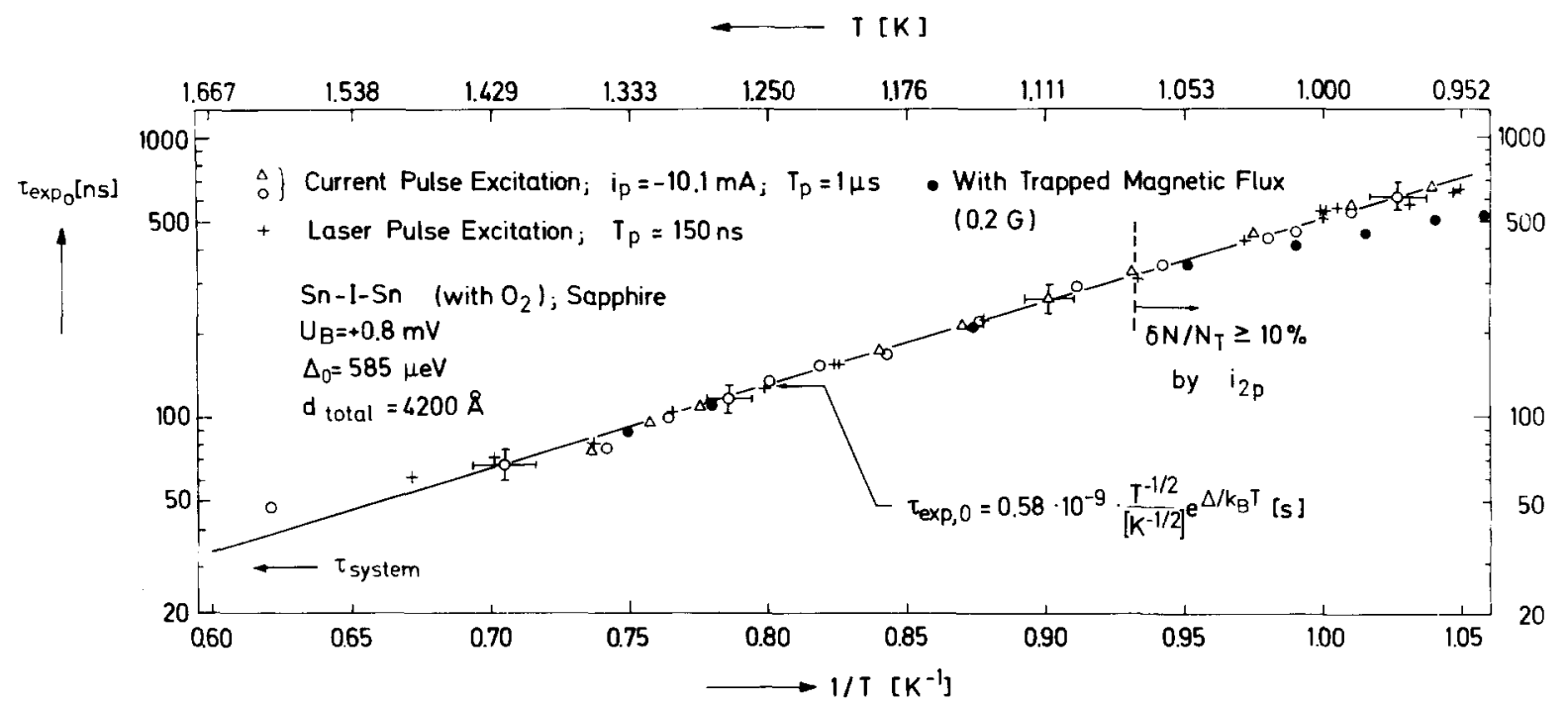

Fig. 5. Experimental quasiparticle recombination lifetimes $\tau_{\text {exp, }, 0}$ versus $1 / T$ for a superconducting tin-tunnel junction immersed in superfluid He. Overinjection by the "two-particle current" at the biasing point $U_{B}=0.8 \mathrm{mV}>A / e$ has been taken into account

Sn [17]:

$\tau_{\text {exp }, 0}=d \frac{N_{\mathrm{th}}}{N_{\omega, \text { th }}}\left(\frac{1}{c_{t}^{3}}+\frac{1}{2 c_{l}^{3}}\right) /\left(\frac{\bar{T}_{t}}{c_{t}^{2}}+\frac{\bar{T}_{l}}{2 c_{l}^{2}}\right)$

where $\bar{T}_{t, l}=\bar{T}_{t, l ; F / S}+\bar{T}_{t, l ; F / \mathrm{He}}$ the total average phonon transmission at the boundaries film $(F)$ /substrate $(S)$ and film/helium with

$\bar{T}_{t, l}=\int_{0}^{\pi / 2} T_{t, l}(\theta) \sin 2 \theta d \theta$,

the average value of the angle and polarization dependent phonon transmission coefficient $T_{t, l}(\theta)$. As the transverse phonon contribution to the transmission across the junction boundaries is dominant (appr. 85\%), it follows from (20)

$\tau_{\text {exp }, 0}=d \frac{N_{\mathrm{th}}}{N_{\omega, \mathrm{th}}} / c\left(\bar{T}_{F / \mathrm{S}}+\bar{T}_{F / \mathrm{He}}\right)$.

With the experimental data for $N_{0}$ and $\Delta_{0}$ listed in Table 1 as well as the transverse sound velocity $c$ $=1.67 \cdot 10^{5}[\mathrm{~cm} / \mathrm{s}][17]$ and the relation $c_{t} \simeq 2 c_{t}$ we obtain from (14) in good approximation:

$\frac{N_{\mathrm{ih}}}{N_{\omega, \text { th }}}=1.56 \frac{T^{-1 / 2}}{\left[K^{-1 / 2}\right]} \exp \left(\Delta_{0} / k_{B} T\right)$

by taking into account only the $Z^{1 / 2}$ term. Applying Equation (22) to the specified data and using $\bar{T}_{\mathrm{Sn} / \mathrm{Al}_{2} \mathrm{O}_{3}}$ $=0.07$ (from acoustic mismatch model) [17] and $\bar{T}_{\mathrm{Sn} / \mathrm{He}}=0.32$ (from $\tau_{\exp }$-measurements in vacuum and He) [2] or $\bar{T}_{\mathrm{Sn} / \mathrm{He}}=0.5$ (from Kapitza resistance) (Ref. 8 in [5]) results in

$\tau_{0}=1.0 \mathrm{~ns}$ or $0.7 \mathrm{~ns}$, respectively.

In order to check this result we also measured the lifetimes in oxygen-free Sn-junctions (evaporated on sapphire in a vacuum of a residual pressure of
$10^{-6}$ Torr), the other parameters, as for instance the contact to liquid $\mathrm{He}$, being unchanged. Within the measurement accuracy there was no deviation compared to those in Figure 5. Therefore, the difference between the experimental (19) and calculated result (24) may not be ascribed to volume losses of $2 \Delta$ phonons in the oxygen-doped tin samples or an enhanced $2 \Delta$-phonon escape by diffuse phonon scattering at the junction boundaries. This is consistent with the data in Table 1 obtained for $\mathrm{O}_{2}$-free and $\mathrm{O}_{2}-$ doped tin films. In view of the uncertainty involved in determining $\bar{T}_{\mathrm{Sn} / \mathrm{He}}$ the agreement between experiment and calculation seems to be good.

Experimental lifetime values in tin samples, evaporated, however, on silicon substrates turned out to be lower by approximately $10 \%$. This reduction is within the measuring error, however can be fully accounted for the changed phonon transmission at the boundary tin/substrate.

Finally we have calculated a mean value $N_{0}$ for the density of electronic states at the Fermi level (presented in Table 1 with its plus - minus standard deviation) from Equation (5) and with data of the analyzed measurements on three superconducting tunnel junctions at then different temperatures in each case. The maximum scatter of the single $N_{0}$ values relating to the mean value amounts to $10 \%$. From Equation (5) we estimated a maximal uncertainty for $N_{0}$ of $30 \%$ to $40 \%$ corresponding to the main sources of error: $\tau_{\text {exp }, 0}( \pm 5 \%), V_{T u}( \pm 5 \%), x(0)$ $( \pm 10 \%)$ and $\Delta( \pm 2 \%)$; the last-quoted results in an error of $12 \%$ in $\exp \left(\Delta / k_{B} T\right)$ at $\Delta=6 k_{B} T$. The presented value for $N_{0}$ from our direct quasiparticle lifetime experiments on thin, superconducting tin films of polycrystalline structure is to be compared 
Table 1. Some characteristic parameters of tin films evaporated in a vacuum at a residual pressure of $10^{-6}$ Torr and/or a pressure of $8 \cdot 10^{-6}$ Torr oxygen. $\rho_{4.2}$ = resistivity measured at $T=4.2 \mathrm{~K}, R=$ film resistance

\begin{tabular}{|c|c|c|c|c|c|c|c|c|c|}
\hline $\begin{array}{c}\Delta_{0} \\
{[\mu \mathrm{eV}]}\end{array}$ & $\begin{array}{c}\mathrm{T}_{\mathrm{C}} \\
{[\mathrm{K}]}\end{array}$ & $\begin{array}{r}2 \Delta_{0} \prime \\
k_{B} T_{C}\end{array}$ & $\begin{array}{l}\delta \mathrm{T}_{\mathrm{C}} \\
{[\mathrm{K}]}\end{array}$ & $\begin{array}{l}d \\
{[\&]}\end{array}$ & $\begin{array}{c}24.2 \\
\left(10^{-7} \Omega \mathrm{cm}\right)\end{array}$ & $\begin{array}{l}I_{4.2} \\
{[\AA]}\end{array}$ & $\left.\begin{array}{c}\xi \\
{[\AA]}\end{array}\right]$ & $\begin{array}{l}R_{4.2} \prime \\
R_{300}\end{array}$ & $\begin{array}{c}\overline{\mathrm{N}_{0}{ }^{\mathrm{H}}} \\
{\left[10^{22} \mathrm{eV}^{-1} \mathrm{~cm}^{-3}\right]}\end{array}$ \\
\hline 585 & 3.76 & 3.61 & 0.08 & 2000 & 6.2 & 1700 & $\begin{array}{l}2000 \\
\text { at } 1 \mathrm{~K}\end{array}$ & 0.053 & $2.73 \pm 0.03$ \\
\hline \multicolumn{10}{|c|}{$\begin{array}{l}\text { bulk value } \mathrm{N}_{0}{ }^{4 t}=2.77 \cdot 10^{22} \mathrm{eV}^{-1} \mathrm{~cm}^{-3} \\
\text { from specific-heat measurements }\end{array}$} \\
\hline
\end{tabular}

with the value determined from conventional measurements $[23,24]$ of the electronic specific heat on ordinary bulk tin. The electronic heat capacity coefficient is proportional to the band-structure density of states $N_{0}^{b s}$ times the enhancement factor $(1+\lambda)$ due to the electron - phonon coupling $\lambda$ [25].

As Table 1 shows, there is an excellent agreement between the film and bulk values $N_{0}$ within the given limits of error. This result is compatible with other film properties we have also measured and included in Table 1. Thus, $T_{c}$ (film) is only enhanced to $1 \%$ compared to $T_{\mathrm{c}}$ (bulk); for comparison we evaluated from the BCS equation for $T_{c}$ an enhancement of $T_{c}$ of $43 \%$ by varying exclusively $N_{0}$ to $10 \%$. Moreover, the coherence length $\xi$ at $T=1 \mathrm{~K}$ is of the order of the film thickness $d$ and the mean free path $l_{4.2}$ (at $4.2 \mathrm{~K}$ ) of electrons due to elastic scattering at defects, internal and external film boundaries; $l_{4.2}$ gives approximately a lower limiting value for the average grain size $\bar{D}$ in our tin films.

After testing the reliability of the described method on superconducting tin tunnel junctions we have also applied it to aluminium and lead samples with different aspects in each case [12]. By experiments on Al we wanted to answer the question, whether the measured $T_{c}$ enhancement of oxygen-perturbed, granular Al films may be caused by a change in $N_{0}$. Furthermore, we checked whether the effective quasiparticle recombination lifetimes are changed with increasing perturbations of the film structure. Measurements on high-quality lead junctions under vacuum conditions were made in order to draw conclusions from the effect of inelastic $2 \Delta$-phonon bulk loss processes on the value of $\tau_{\exp , 0}$. A detailed description of these experiments will be published separately.

\section{References}

1. Eisenmenger, W.: in: Tunneling Phenomena in Solids, E. Burstein and S. Lundquist (eds.), p. 371. New York: Plenum Press 1969
2. Trumpp, H.J., Lassmann, K., Eisenmenger, W.: Phys. Lett 41 A, 431 (1972)

3. Parker, W.H., Williams, W.D.: Phys. Rev, Lett. 29, 924 (1972)

4. Sai-Halasz, G.A., Chi, C.C., Denenstein, A., Langenberg, D.N.: Phys. Rev. Lett. 33, 215 (1974)

5. Parker, W.H.: Sol. State Comm. 15, 1003 (1974)

6. Hu, P., Dynes, R.C., Narayanamurti, V.: Phys. Rev. B10, 2786 (1974)

7. Ginsberg, D.M.: Phys. Rev. Lett. 8, 204 (1962)

8. Miller, B.I., Dayem, A.H.: Phys. Rev. Lett. 18, 1000 (1967)

9. Levine, J.L., Hsieh, S.Y.: Phys. Rev. Lett. 20, 994 (1968)

10. Gray, K.E., Long, A.R., Adkins, C.J.: Phil. Mag. 20, 273 (1969)

11. Sonnenberg, K.H.: Diplomarbeit, Universität Göttingen (1970), unpublished

12. Epperlein, P.W.: Thesis, Universität Stuttgart (1977). Quasiparticle recombination lifetime measurements on a single superconducting tunnel junction by current pulse excitation were made for the first time by [10] and [11]. The requirements for the pulse electronics, however, increase in the sequence of experiments on $\mathrm{Al}[10,12]$, Sn $[11,12]$ and $\mathrm{Pb}[12]$ because of the corresponding, decreasing lifetimes.

13. Gray, K.E.: J. Phys. F (Metal Phys.) 1, 290 (1971)

14. Long, A.R.: J. Phys. F (Metal Phys.) 3, 2040 (1973)

15. Smith, L.N., Mochel, J.M.: Phys. Rev. Lett. 35, 1597 (1975)

16. Rothwarf, A., Taylor, B.N.: Phys. Rev. Lett. 19, 27 (1967)

17. Eisenmenger, W., Lassmann, K., Trumpp, H.J., Krauss, R.: Appl. Phys. 11, 307 (1976); Appl. Phys. 12, 163 (1977)

18. Eisenmenger, W.: in: Physical Acoustics Vol. XII, 1976 (Eds. W.P. Mason and R. Thurston)

19. Schrieffer, J.R., Wilkins, J.W.: Phys. Rev. Lett. 10, 17 (1963)

20. Schuller, I., Gray, K.E.: Phys. Rev. B12, 2629 (1975)

21. Zappe, H.H., Grebe, K.R.: J. Appl. Phys. 44, 865 (1973)

22. Scott, W.C.: Appl. Phys. Lett. 17, 166 (1970)

23. Kitte1, C.: Introduction to Solid State Physics, IIIrd Edition. New York: J. Wiley \& Sons 1967

24. Gopal, E.S.R.: Specific Heats of Low Temperatures, London: Plenum Press 1966. K. Mendelssohn and K.D. Timmerhaus (eds.) The bulk value for $N_{0}$ listed in Table 1 was evaluated from the relation $N_{0}=3 \gamma / \pi^{2} k_{B}^{2}$ with an average electronic specific heat coefficient $\gamma$, obtained from [23] and [24].

25. McMillan, W.L.: Phys. Rev. 167, 331 (1968)

P.W. Epperlein

K. Lassmann

W. Eisenmenger

Physikalisches Institut

Universität Stuttgart

Pfaffenwaldring 57

D-7000 Stuttgart 80

Federal Republic of Germany 